\title{
Accurate setting of electron energy for demonstration of first hadron beam cooling with rf-accelerated electron bunches
}

S. Seletskiy®, M. Blaskiewicz, A. Drees $\odot$, A. Fedotov®, W. Fischer, D. M. Gassner®, X. Gu,

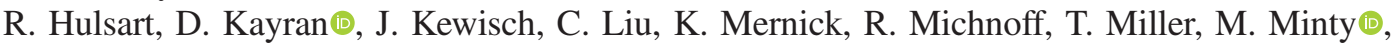
G. Robert-Demolaize, V. Schoefer, H. Song, P. Thieberger $\odot$, and P. Wanderer®

Brookhaven National Laboratory, Upton, New York 11973, USA

(Received 21 October 2019; published 27 November 2019)

\begin{abstract}
The world's first electron cooling based on the rf acceleration of electron bunches was experimentally demonstrated at the Low Energy RHIC Electron Cooler (LEReC) at Brookhaven National Laboratory. The critical step in obtaining cooling of the Au ions in the collider with this new approach was matching the electron and ion relativistic $\gamma$-factors with a relative error of less than $5 \times 10^{-4}$. Since the electron beam kinetic energy was just $1.6 \mathrm{MeV}$, it was required to set the absolute energy of electrons with an accuracy better than $0.8 \mathrm{keV}$. The method of setting electron energy in conventional coolers was unsuitable for LEReC and a new technique had to be developed. In this paper we describe our experience with measuring the electron beam energy at LEReC and precisely matching electron and ion $\gamma$-factors, which resulted in demonstration of the cooling.
\end{abstract}

DOI: 10.1103/PhysRevAccelBeams.22.111004

\section{INTRODUCTION}

The phase space density of ion bunches in the collider decreases with time due to intrabeam scattering (IBS) and due to the lack of radiation damping. This results in an unwanted reduction of integrated luminosity of the collider. To counteract this process the "cooling" (either electron [1] or stochastic [2]) of the ion bunches is desired. While stochastic cooling was successfully implemented at the Relativistic Heavy Ion Collider (RHIC) [3], electron cooling at collision energy has never been implemented at any collider until now [4].

Electron cooling, a method of increasing phase space density of heavy particles through their interaction with copropagating "cold" electrons, was introduced by Budker in 1967 and demonstrated in 1974 [5] with nonrelativistic protons. Since then, this method was implemented at numerous nonrelativistic (with Lorentz $\gamma \lesssim 1.5$ ) proton and ion storage-rings [6].

In a typical nonrelativistic electron cooler a DC electron beam is generated by a thermionic gun and transported in a strong continuous solenoidal magnetic field to the cooling section (CS), a straight section of the storage ring where electrons propagate together with ions at the same average velocity. The ions interact with electrons in the CS through

Published by the American Physical Society under the terms of the Creative Commons Attribution 4.0 International license. Further distribution of this work must maintain attribution to the author(s) and the published article's title, journal citation, and DOI.
Coulomb scattering, which results in an average friction force that over many revolutions of the ions in the accelerator reduces the ion bunch temperature. The electrons after each passage are either dumped or returned to the gun for charge recovery, thus, on each turn the ions interact with fresh "low temperature" electrons.

Relativistic electron cooling was first demonstrated at Fermilab in 2005 [7,8]. The Fermilab electron cooler was used for longitudinal cooling of $8.9 \mathrm{GeV}$ antiprotons in the storage ring prior to their transfer to the Tevatron collider and featured a 4.3 MeV DC electron beam $[9,10]$.

Applying electron cooling at much higher ion beam energies in future colliders [11], for example in a proposed electron-ion collider [12], requires a new approach. Accelerating DC electron beam to energies higher than $\approx 10 \mathrm{MeV}$ is not practical, therefore a rf accelerator and bunched electron beam must be used for high energy coolers. Also, to counteract the IBS in the collider, the electron cooling must be applied directly to the colliding ions, rather than being used in a pre-injection storage ring. All these novel techniques were successfully realized in the LEReC project.

In any electron cooler a critical step in obtaining the cooling is the precise matching of electron and hadron Lorentz $\gamma$-factors.

In nonrelativistic coolers the electron energy can be measured with $10^{-4}$ accuracy by measuring the gun cathode high voltage with a high-precision voltage divider [13]. Such a method is not available in LEReC.

To set electron energy in the relativistic electron cooler at Fermilab observation of the Schottky spectrum of the coasting hadron beam filling the entire momentum acceptance 
was used [8]. This technique is unsuitable for LEReC because of the electron-hadron heating [14], which is prohibitively high for a coasting (hence, not locked in rf frequency to electron bunches) ion beam.

Therefore, for setting of the electron energy in LEReC we employed a combination of a state of the art highaccuracy low energy spectrometer and a hadron-electron recombination monitor with electron energy scan.

\section{LEReC ACCELERATOR}

LEReC is a crucial part of RHIC operation dedicated to the search for the QCD critical point in the nuclear matter phase diagram [15]. LEReC was designed and built to compensate the IBS-driven emittance growth of colliding ion bunches with relativistic factors $\gamma=4.1,4.9$ and 6.1, which correspond to electron beam kinetic energies of 1.6 MeV, $2 \mathrm{MeV}$ and 2.6 MeV respectively. While the Fermilab electron cooler approach could have been used at these energies, it was decided to build an electron cooler that has all the features required for future high energy electron coolers.

The LEReC accelerator [16], shown in Fig. 1, consists of a $400 \mathrm{keV}$ photo-gun [17] followed by a $704 \mathrm{MHz}$ superconducting rf accelerating cavity (SRF Booster) [18], which accelerates the beam to $1.6-2.6 \mathrm{MeV}$. The photocathode is illuminated by a green $704 \mathrm{MHz}$ laser modulated with the $9 \mathrm{MHz}$ frequency to match the frequency of RHIC ions. The resulting $9 \mathrm{MHz}$ "macro-bunches" of electrons consist of thirty $704 \mathrm{MHz}$ bunches each.

Electron bunches are accelerated in the SRF cavity offcrest producing an energy-time dependence along each bunch (a "chirp"). The chirped bunch is ballistically stretched via time-of-flight dispersion in the transport beamline. Hence, the space charge effect on the bunch energy spread and emittance are substantially reduced. The SRF Booster is followed by a 3rd harmonic cavity which linearizes the longitudinal phase space of the bunch.
There is another $704 \mathrm{MHz}$ cavity at the end of the transport beamline to remove the energy chirp. The $9 \mathrm{MHz}$ rf cavity is used to reduce the effect of beam-loading over the length of the $9 \mathrm{MHz}$ macro-bunch.

The transport beamline and the merger bring the electron beam to the two cooling sections [19] in the "yellow" and in the "blue" RHIC rings which are connected by a $180^{\circ}$ bending magnet. The Blue CS is followed by the extraction to the beam dump.

LEReC is a nonmagnetized electron cooler-it does not utilize strong magnetic field in the cooling section. The short CS solenoids (eight solenoids per cooling section) are used only for correction of the beam envelope [20].

\section{REQUIREMENTS FOR THE ELECTRON BEAM}

The friction force acting on the ion with a charge $Z e$, where $e$ is the electron charge, due to its interaction with a nonmagnetized electron bunch with velocity distribution $f\left(v_{e}\right)$ is given by [21,22]:

$$
\vec{F}=-\frac{4 \pi n_{e} e^{4} Z^{2}}{m_{e}} \int L_{C} \frac{\vec{v}_{i}-\vec{v}_{e}}{\left|\vec{v}_{i}-\vec{v}_{e}\right|^{3}} f\left(v_{e}\right) d^{3} v_{e} .
$$

Here, $n_{e}$ is the electron bunch density in the beam frame, $m_{e}$ is the mass of the electron, $\vec{v}_{i}$ and $\vec{v}_{e}$ are ion and electron velocities in the beam frame. Coulomb logarithm is $L_{C}=$ $\ln \left(\rho_{\max } / \rho_{\min }\right)$ with a minimal impact parameter

$$
\rho_{\min }=\frac{Z e^{2}}{m_{e}} \frac{1}{\left|\vec{v}_{i}-\vec{v}_{e}\right|^{2}}
$$

At a given value of the ion velocity $\rho_{\max }=$ const and is determined by the ion time of flight through the CS.

The Coulomb logarithm can be assumed to be constant, in the LEReC case $L_{C} \approx 10$. We assume a Gaussian distribution of velocities in the electron bunch with root mean square (rms) values $\Delta_{t}=\sqrt{\Delta_{x}^{2}+\Delta_{y}^{2}}$ and

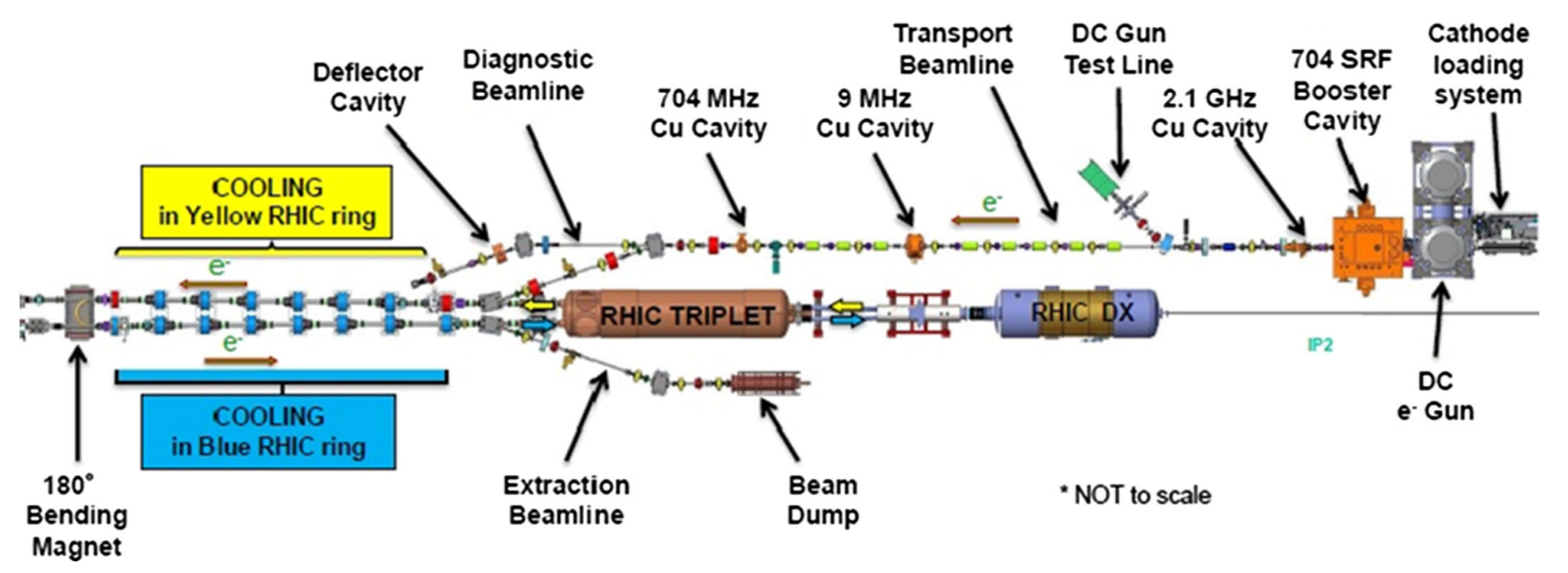

FIG. 1. LEReC layout (not to scale). 
$\Delta_{z}$ for transverse and longitudinal velocity components $\left(v_{e t}=\sqrt{v_{e x}^{2}+v_{e y}^{2}}\right.$ and $\left.v_{e z}\right)$ respectively:

$f\left(v_{e}\right)=\frac{1}{(2 \pi)^{3 / 2} \Delta_{x} \Delta_{y} \Delta_{z}} \exp \left(-\frac{v_{e x}^{2}}{2 \Delta_{x}^{2}}-\frac{v_{e y}^{2}}{2 \Delta_{y}^{2}}-\frac{v_{e z}^{2}}{2 \Delta_{z}^{2}}\right)$.

LEReC was designed to cool ion bunches with an expected rms longitudinal and transverse velocity spread (in the beam frame) of $1.5 \times 10^{5} \mathrm{~m} / \mathrm{s}$ and $1.7 \times 10^{5} \mathrm{~m} / \mathrm{s}$ respectively.

The dependence of the longitudinal friction force on the ion velocity for any given $\Delta_{z}$ is shown in Fig. 2 .

As one can see, the friction force is maximized for the longitudinal ion velocity $v_{i z}=1.5 \Delta_{z}$ and it is linear for $v_{i z} \lesssim \Delta_{z}$. Since the cooling under discussion is directly applied to the ions in the collider, it is important not to overcool the ion bunch. Therefore, the requirement for the rms spread of the electron bunch longitudinal velocities is $\Delta_{z} \approx\left(\overline{v_{i z}^{2}}\right)^{1 / 2} \approx 1.5 \times 10^{5} \mathrm{~m} / \mathrm{s}$. From similar considerations $\Delta_{t} \approx 1.7 \times 10^{5} \mathrm{~m} / \mathrm{s}$. Converting the longitudinal and transverse velocity spreads in the beam frame into relative energy spread $\left(\sigma_{\delta}\right)$ and angular spread $\left(\sigma_{\theta}\right)$ in laboratory frame respectively, we obtain the following requirements for LEReC electron bunch parameters [20]:

$$
\sigma_{\delta}=\frac{\Delta_{z}}{\beta c}=5 \times 10^{-4} ; \quad \sigma_{\theta}=\frac{\Delta_{t}}{\gamma \beta c}=150 \mu \mathrm{rad}
$$

Since the average velocity of the two beams must be matched to better than the respective rms velocity spread, the relativistic $\gamma$-factors of ion and electron bunches also must be matched with an accuracy of better than $5 \times 10^{-4}$.

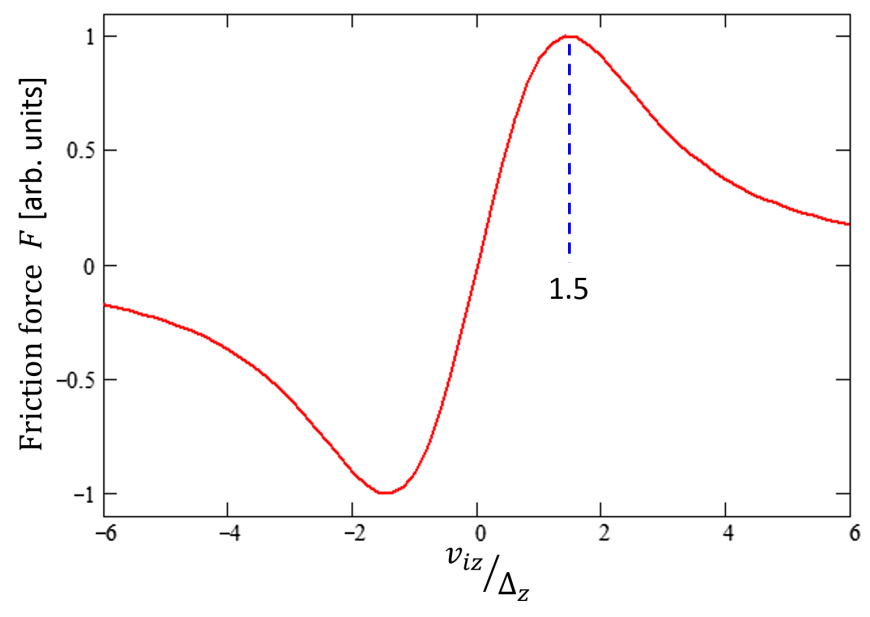

FIG. 2. Normalized longitudinal friction force depending on the ratio of longitudinal ion velocity to the rms spread of velocities in the electron bunch. The force is plotted for an ion with its transverse velocity equal to the rms spread of transverse ion velocities.

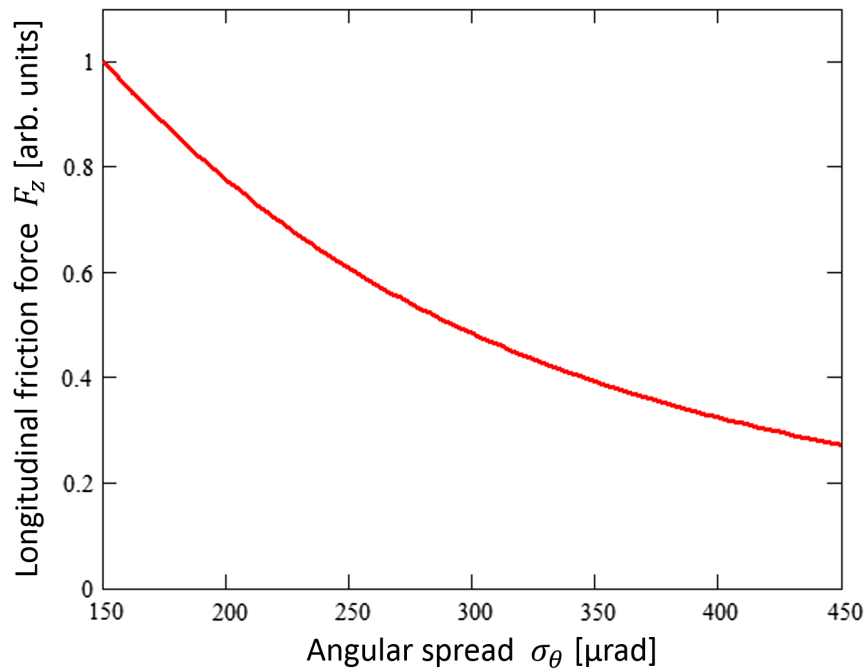

FIG. 3. Normalized longitudinal friction force depending on the rms angular spread in an electron bunch. Equation (1) is integrated for an ion with $v_{i}=\sqrt{\bar{v}_{i}^{2}}$. It is assumed that $\sigma_{\delta}=5 \times 10^{-4}$.

Electron bunches satisfying requirements (4) were obtained during LEReC accelerator commissioning in 2018 [16].

As Fig. 3 illustrates, the longitudinal friction force has a relatively weak dependence on electron angular spread, which is affected by the presence of the ion beam in the CS [23]. Since the electron bunch momentum spread is satisfying Eq. (4) regardless of the presence of ions, it shall be sufficient to match the electron and ion beam $\gamma$-factors to observe longitudinal cooling.

\section{MATCHING OF $\gamma$-FACTORS}

The absolute energy of the electron and ion beam must be known with high accuracy to match the electron and ion beam $\gamma$-factors. The absolute energy of ions in the collider is known with an accuracy of better than $10^{-4}$. On the other hand, the absolute energy of the electron beam derived from settings of the rf cavities can have an error of a few percent. Without a beam based calibration, it is difficult to achieve an accuracy of better than a few percent in the rf cavity field probe calibration, limited by the intrinsic calibration measurement errors in the field probe coupling factor, rf power, and cable losses.

Our approach for accurately setting the electron beam energy was three-fold: (1) First, we used a dedicated low energy spectrometer to set the energy to a $5 \times 10^{-3}$ accuracy [24]. (2) Second, we roughly matched the electron and ion beam $\gamma$-factors by scanning the energy of the electron beam in small steps and measuring the rate of ionelectron recombination [25]. The maximum recombination rate corresponds to matched $\gamma$-factors. (3) Finally, we performed an electron beam energy scan with finer steps until we observed longitudinal cooling of the ions. 
The $\gamma$-matching was performed sequentially using the outlined techniques. Optimized cooling was obtained for well matched $\gamma$-factors.

\section{A. High-accuracy low energy spectrometer}

The $180^{\circ}$ bending magnet located between the "yellow" and the "blue" RHIC cooling sections was used as a spectrometer. This U-turn bend is designed to have a bending radius of $\rho_{0}=0.35 \mathrm{~m}$. The bend entrance is equipped with two beam position monitors (BPMs), and its exit is equipped with one BPM. The BPM-to-BPM distance and distance between bend exit and entrance to BPMs was a compromise between optimizing the CS optics and the accuracy of energy measurement. The spectrometer setup is shown in Fig. 4.

The design accuracy of the energy spectrometer is $8 \mathrm{keV}$. It is defined by the mechanical alignment of the U-bend magnet and the BPMs assembly, the accuracy of the BPM measurements, the accuracy of the bend field map and the stability of the bend power supply. The detailed account of the factors contributing to the spectrometer accuracy is given in $[24,26]$.

The field of the U-turn bending magnet was mapped in a $\pm 20 \mathrm{~mm}$ range around the nominal beam trajectory at $20 \mathrm{mT}, 24 \mathrm{mT}$, and $30 \mathrm{mT}$ flat-top fields corresponding to 1.6 MeV, $2 \mathrm{MeV}$, and 2.6 MeV electron beam energies respectively [27]. The field was measured with a probe combining a Hall sensor and a unique, customized highaccuracy NMR probe capable of measuring fields as low as $14 \mathrm{mT}$ [28]. The resulting field map achieved the required accuracy of $2 \mu \mathrm{T}$.

After installation of the spectrometer in the RHIC tunnel the same probe was installed at a known, and well-mapped, fixed location in the uniform region of the bending field. The continuous readings of the probe were used for the energy measurement. Carefully optimized

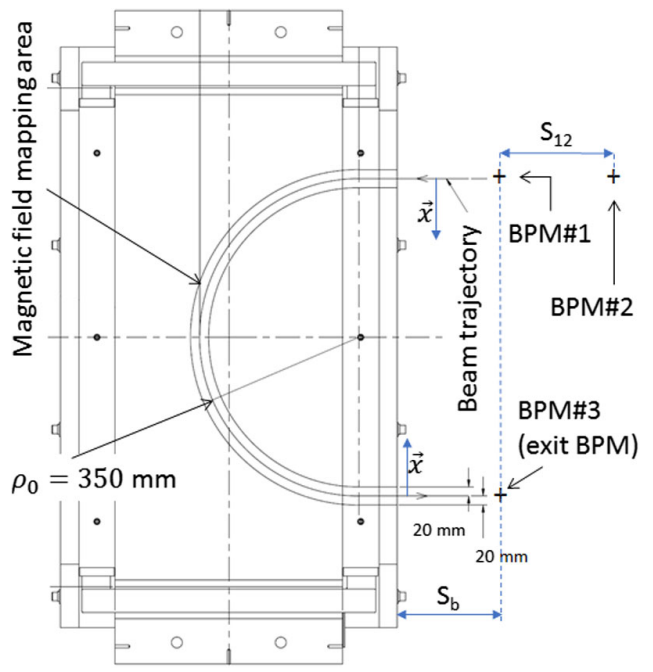

FIG. 4. Layout of LEReC spectrometer. hysteresis cycling for calibrations and for operation was essential to maintain the required field accuracy.

In the hard-edge approximation the horizontal displacement of the electron beam trajectory at the exit of $180^{\circ}$ bend $\left(x_{\text {out }}\right)$ is given by:

$$
x_{\text {out }}=-x_{\text {in }}+2 \rho_{0}-2 \rho \cos \theta_{\text {in }} .
$$

Here $x_{\text {in }}$ and $\theta_{\text {in }}$ are the trajectory horizontal displacement and angle at the entrance to the bend and $\rho$ is the trajectory curvature radius at the actual beam energy $E$. The axis $\vec{x}$ always points "inward" and flips its direction as the beam passes through the $180^{\circ}$ bend.

As described in $[24,26]$, to measure the beam energy in the 1.6-2.6 $\mathrm{MeV}$ range with the accuracy required for LEReC one must perform a proper Taylor expansion of the exact expression for magnetic rigidity. Then, the beam kinetic energy can be calculated from spectrometer readings as:

$E=E_{0}+E_{0} \frac{E_{0}+2 m_{e} c^{2}}{E_{0}+m_{e} c^{2}}\left(\frac{B-B_{0}}{B_{0}}-\frac{B}{B_{0}} \frac{x_{\text {out }}+x_{\text {in }}}{2 \rho_{0}}\right)$

with

$x_{\text {in }}=x_{2}+S_{b} \frac{x_{2}-x_{1}}{S_{12}} ; \quad x_{\text {out }}=x_{3}+S_{b} \frac{x_{2}-x_{1}}{S_{12}}$.

Here, $E_{0}$ is the design beam kinetic energy, $B_{0}$ is the hardedge magnetic field taken from the field mapping data, $x_{1}$, $x_{2}$ and $x_{3}$ are horizontal readings of the two entrance and one exit spectrometer BPMs respectively, $S_{12}$ is the distance between the two entrance BPMs measured during a mechanical survey and $S_{b}$ is the distance between the edge of the bending magnet in hard-edge approximation and the entrance/exit BPMs. The distance $S_{b}$ is calculated from survey and field mapping data.

The result of Eq. (6) was always compared with beam tracking in the mapped field [26] during energy measurements. The results of tracking and calculations were found to be in good agreement.

First energy measurements showed a small but noticeable dependence on the electron beam trajectory through our spectrometer, possibly due to inconsistencies in BPMs calibration. Hence, we performed a dedicated beam-based calibration (BBC) of the spectrometer BPMs with the ion beam.

The $\mathrm{BBC}$ consisted of moving the ion beam trajectory through the CS transversely in the absence of the electron beam in each cooling section and recording the response in the ion position measured by the spectrometer BPMs. Since the ion trajectory in the CS is a straight line, due to the ions large magnetic rigidity, the $\mathrm{BBC}$ allowed us to find the proper BPMs scaling coefficients. After the coefficients were corrected the energy measurement dependence on the beam trajectory became negligible, as Fig. 5 demonstrates. 


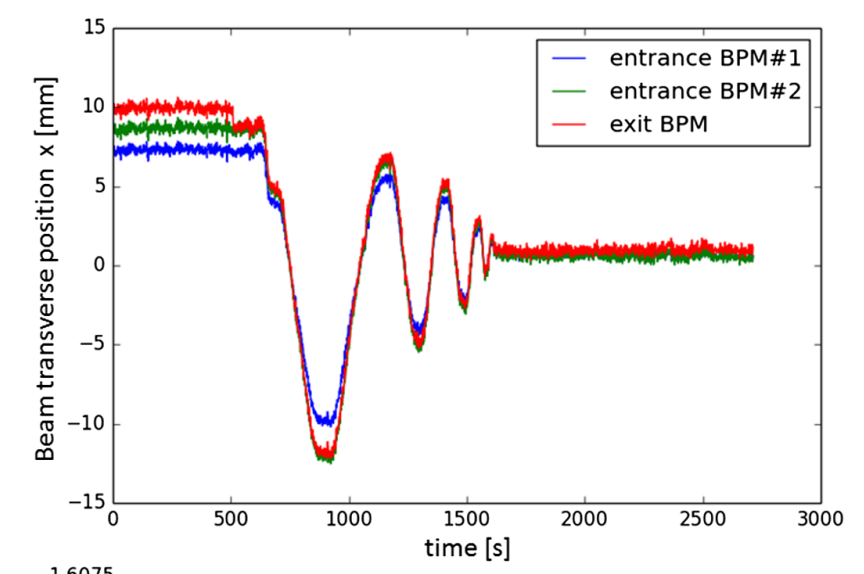

$$
\alpha_{r}=\frac{n_{e} \eta \xi}{(2 \pi)^{\frac{3}{2}} \gamma^{2}} \int \sigma\left(\left|\vec{v}_{i}-\vec{v}_{e}\right|\right) f\left(v_{e}\right) f\left(v_{i}\right) d v_{e}^{3} d v_{i}^{3} .
$$

Here $n_{e}$ is the electron bunch density, $\eta$ is the fraction of RHIC ring circumference occupied by the cooling section, $\xi$ is the duty factor, which includes both the geometrical longitudinal overlap between the thirty electron bunches and the ion bunch and a fraction of ion bunches interacting with the electrons, $f\left(v_{i}\right)$ is the velocity distribution in the ion bunch and the capture cross section $\sigma$ is given by [29]:

$$
\begin{aligned}
\sigma= & \frac{2 A h \nu_{0}}{m_{e}\left|\vec{v}_{i}-\vec{v}_{e}\right|^{2}}\left[\ln \sqrt{\frac{2 h \nu_{0}}{m_{e}\left|\vec{v}_{i}-\vec{v}_{e}\right|^{2}}}+0.1402\right. \\
& \left.+0.525\left(\frac{m_{e}\left|\vec{v}_{i}-\vec{v}_{e}\right|^{2}}{h \nu_{0}}\right)^{\frac{1}{3}}\right]
\end{aligned}
$$

where $h$ is Planck's constant, $h \nu_{0}=13.6 Z^{2} \mathrm{eV}$ is the ground state binding energy and a constant $A=\frac{4 h e^{2}}{\sqrt{27} \pi \epsilon_{0} m_{e}^{2} c^{3}}$.

The cross section, and thus the recombination rate, is the highest when ion and electron beam $\gamma$-factors are equal.

A special lattice (see Fig. 6) was developed with a dispersion bump in a RHIC arc downstream of the cooling section [30]. The losses due to recombination were registered in this region with a dedicated plastic scintillation detector (PSD) [31].

Although the PSD signal was rather noisy, the increase in

FIG. 5. Stable energy measurement during induced change in the electron trajectory. The upper plot shows readings of the two entrance and one exit spectrometer BPMs. The lower plot shows the measured electron beam energy.

Our first goal was to obtain electron cooling of ions with $\gamma=4.1$, which requires electrons with $1.6 \mathrm{MeV}$ energy. While the initial LEReC setting was based on calibration of rf cavities, the spectrometer showed us that our real energy was $100 \mathrm{keV}$ lower than the design energy of $1.6 \mathrm{MeV}$. After retuning the accelerator based on the spectrometer measurements, we were ready for precise $\gamma$-matching.

\section{B. Energy scanning and recombination monitor}

The scan of the electron beam energy must preserve the energy spread of the electron bunch. Adjusting the electron beam energy with the booster cavity while keeping a small energy spread of electron bunches is a complex process. Fortunately, the last "chirp-correcting" cavity is operated at the zero-crossing, so that electron bunches probe the linear part of the sinusoidal wave. Thus, by adjusting the phase of this cavity one can change the electron bunch energy while still satisfying Eq. (4). The cavity voltage is such that the available range for an energy scan is about $\pm 15 \mathrm{keV}$.

An ion $\left(\mathrm{Au}^{79+}\right)$ that recombines with an electron through the radiative recombination process in the cooling section becomes $\mathrm{Au}^{78+}$ and is lost in the high dispersion areas of the RHIC ring. The recombination rate $\left(\alpha_{r}\right)$ is given by [25]: recombination rate during the energy scan was clearly visible and well repeatable. Figure 7 shows the recombination signal from 6 ion bunches overlapped with 6 electron macro-bunches during the energy scan. As one can see, the matched energy was $12 \mathrm{keV}$ lower than the energy set based on the initial spectrometer readings.

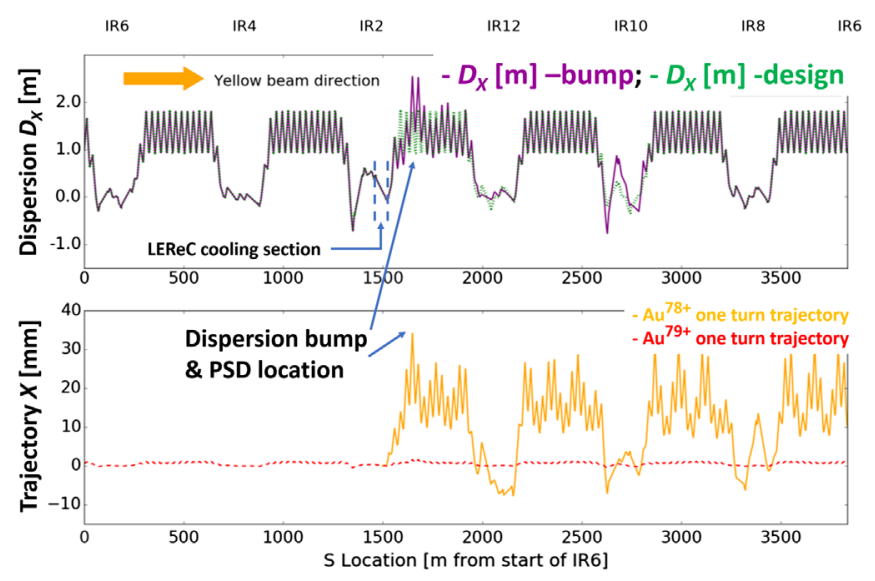

FIG. 6. The upper plot shows the dispersion in a design RHIC lattice (green dotted line) and in a "dispersion bump" lattice (violet solid line). The lower plot shows one turn horizontal trajectory of the recombined $\mathrm{Au}^{78+}$ ion (orange solid line) and the trajectory of the $\mathrm{Au}^{79+}$ ion (red dotted line) in the dispersion bump lattice. 


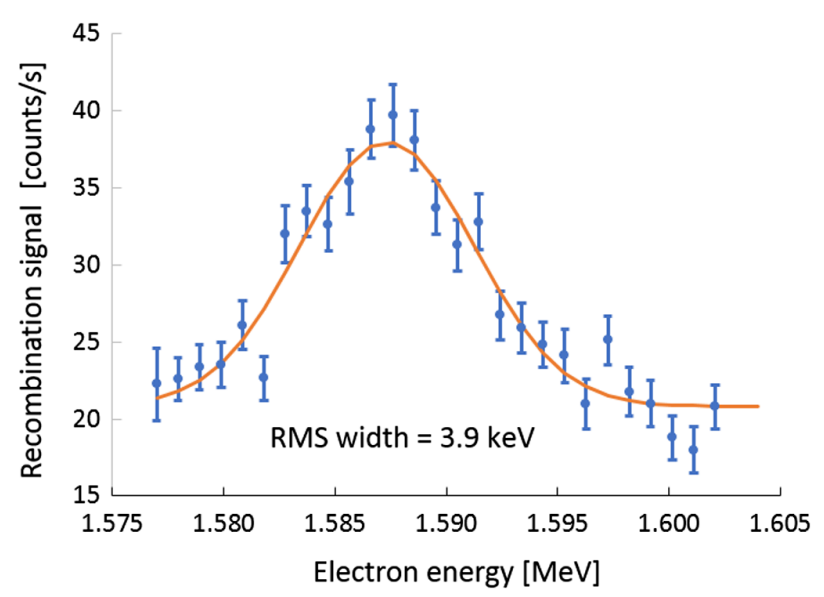

FIG. 7. The recombination signal (blue dots) dependence on beam energy (as measured by spectrometer) during the scan. The error bars for each point represent the rms of the recombination signal at each energy setting averaged over two scans. RMS width of the measured trend is calculated from Gaussian fit of the data (orange solid line).

The "recombination energy scan" was performed before we determined the reason for and corrected the spectrometer measurement dependence on electron beam trajectory. After the correction was done the error of spectrometer measurement became $6 \mathrm{keV}$, as Fig. 5 demonstrates. Thus, the accuracy of the energy spectrometer reached $4 \times 10^{-3}$.

\section{Demonstration of longitudinal cooling}

In the absence of cooling, the length of the ion bunches increases during a store due to IBS until the ions fill the entire rf bucket. With electron cooling on, the length of the ion bunch must stabilize at some smaller value. Therefore, to observe longitudinal cooling we injected six consecutive ion bunches into the RHIC ring and overlapped five of them with the electron bunches. Then we performed an electron beam energy scan in small steps around the value found in recombination measurements. The ion bunch length and shape were continuously monitored during the scan with the RHIC wall current monitor (WCM).

The $9 \mathrm{MHz}$ cavity compensates the beam loading over the length of one macrobunch only. The transient macrobunch to macrobunch beam loading, which is present when LEReC runs with just a few out of the operational 120 macrobunches per RHIC revolution, stays uncompensated. By choosing a train of five electron macrobunches for the energy scan we used this effect to our advantage. Due to beam loading, every subsequent electron macrobunch is shifted in energy with respect to the previous one. For $60 \mathrm{pC}$ per bunch and 30 bunches per macrobunch the measured energy shift was $1 \mathrm{keV}$. Therefore, the train of 5 macrobunches covers about 6 times wider energy range than a single macrobunch with the design rms energy spread of $0.8 \mathrm{keV}$. Such a setup significantly accelerates the energy scan and makes it easier to obtain a $\gamma$-match for one of the ion bunches.

The results of the longitudinal bunched electron cooling are given in Fig. 8. When electron and ion $\gamma$-factors are getting matched the length of the cooled ion bunch starts to shrink very fast as compared to the length of the noninteracting ("test") ion bunch. After several minutes of cooling the cooled ion bunch is much shorter and has a much higher peak current than the test ion bunch.

The optimized electron beam energy was within $1 \mathrm{keV}$ of the matched- $\gamma$ energy measured by the recombination monitor.

It also became clear that the longitudinal cooling itself is a very sensitive monitor for $\gamma$-matching. This allowed us to proceed with a nominal ion beam lattice without a large dispersion bump required for the recombination monitor.

For $\gamma$-matching at $\gamma=4.9$ we used our spectrometer and the energy scanning with ion bunch length monitoring to find the optimal $2 \mathrm{MeV}$ settings for the electron beam. The whole process took just a few hours from rough rf retuning
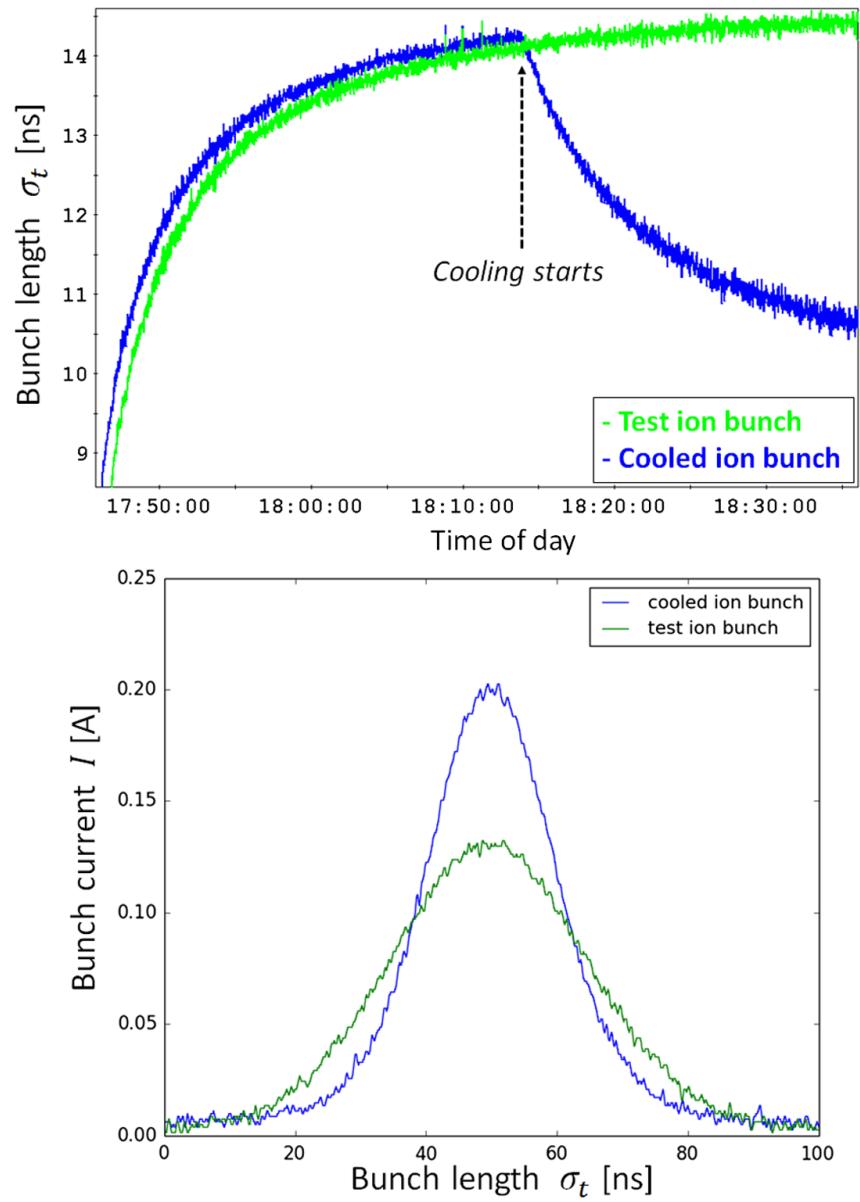

FIG. 8. The typical bunched beam cooling. The upper plot shows evolution of the rms length of a cooled bunch and a test ion bunch. The lower plot shows the longitudinal profiles of a cooled and a test ion bunch after 20 min of cooling. 
to obtaining optimized bunched beam cooling at the new energy.

\section{CONCLUSION}

LEReC is the first rf-based (bunched) electron cooler. It is the first electron cooler applied directly to the ions in a collider at the colliding energy. It is also the first cooler that utilizes the same electron beam to cool ions in two separate rings.

The critical part of obtaining electron cooling in LEReC was setting the electron beam energy with an accuracy of better than $0.8 \mathrm{keV}$ to match electron and ion beam relativistic $\gamma$-factors.

The electron energy setting methods used in conventional DC coolers rely on accurate measurement of DC gun voltage. In the LEReC case the rf accelerated electron bunches have a large energy uncertainty. Therefore, to achieve our goal we developed a high-accuracy low energy spectrometer and used it in conjunction with the recombination monitor and electron energy scan preserving the energy spread of the electron bunch.

These tools allowed us to achieve the required accuracy of energy measurement and $\gamma$-matching, resulting in the attainment of hadron beam cooling with rf-accelerated electron bunches.

\section{ACKNOWLEDGMENTS}

The reported results could not be achieved without dedicated work of the entire staff of the BNL ColliderAccelerator Department. We are grateful to Thomas Roser for his support and encouragement. This work was supported by the U.S. Department of Energy under Contract No. DE-SC0012704.

[1] G. I. Budker, An effective method of damping particle oscillations in proton and antiproton storage rings, At. Energy 22, 346 (1967) [Sov. At. Energy 22, 438 (1967)].

[2] S. van der Meer, Stochastic cooling and the accumulation of antiprotons, Rev. Mod. Phys. 57, 689 (1985).

[3] M. Blaskiewicz, J. M. Brennan, and F. Severino, Operational Stochastic Cooling in the Relativistic Heavy-Ion Collider, Phys. Rev. Lett. 100, 174802 (2008).

[4] A. Fedotov et al., First electron cooling of hadron beams using a bunched electron beam, in Proceedings of NAPAC2019, Lansing, MI, 2019 (to be published).

[5] G. I. Budker et al., Experimental study of electron cooling, Part. Accel. 7, 197 (1976).

[6] H. Poth, Applications of electron cooling in atomic, nuclear and high-energy physics, Nature (London) 345, 399 (1990).

[7] S. Nagaitsev et al., Experimental Demonstration of Relativistic Electron Cooling, Phys. Rev. Lett. 96, 044801 (2006).

[8] S. Seletskiy, Ph.D. thesis, University of Rochester, 2005.
[9] A. Shemyakin et al., Attainment of an MeV-range, DC electron beam for the Fermilab cooler, Nucl. Instrum. Methods Phys. Res., Sect. A 532, 403 (2004).

[10] A. Burov, S. Nagaitsev, A. Shemyakin, and Y. Derbenev, Optical principles of beam transport for relativistic electron cooling, Phys. Rev. Accel. Beams 3, 094002 (2000).

[11] A. Fedotov, I. Ben-Zvi, D. L. Bruhwiler, V. N. Litvinenko, and A. O. Sidorin, High-energy electron cooling in a collider, New J. Phys. 8, 283 (2006).

[12] C. Montag, eRHIC design overview, in Proceedings of IPAC2019, Melbourne, Australia (JACoW Publishing, 2019).

[13] H. Poth, Electron cooling: Theory, experiment, application, CERN Report No. CERN-EP/90-04, 1990.

[14] M. Blaskiewicz and J. Kewisch, Emittance growth generated by bunched beam electron cooling, BNL Report No. BNL-211519-2019-TECH, 2019.

[15] K. Rajagopal, Can we discover the QCD critical point at RHIC?, Proc. of RBRC Workshop, V. 80, 2006 [BNL Report No. BNL-75692-2006, 2006].

[16] D. Kayran et al., First results from commissioning of low energy RHIC electron cooler (LEReC), in Proceedings of IPAC2019, Melbourne, Australia (JACoW Publishing, 2019).

[17] X. Gu et al., Operational experience of high-voltage highcurrent gun for LEReC (to be published).

[18] B. Xiao et al., Design and test of $704 \mathrm{MHz}$ and $2.1 \mathrm{GHz}$ normal conducting cavities for low energy RHIC electron cooler, Phys. Rev. Accel. Beams 22, 030101 (2019).

[19] S. Seletskiy et al., Alignment of electron and ion beam trajectories in non-magnetized electron cooler, in Proceedings of IPAC2017, Copenhagen, Denmark (JACoW Publishing, 2017).

[20] A. Fedotov et al., Accelerator physics design requirements and challenges of RF based electron cooler LEReC, in Proceedings of NAPAC16, Chicago, Illinois, USA (JACoW Publishing, 2016).

[21] S. Chandrasekhar, Principles of Stellar Dynamics (University of Chicago Press, Chicago, 1942).

[22] Ya. S. Derbenev and A. N. Skrinsky, The effect of an accompanying magnetic field on electron cooling, Part. Accel. 8, 235 (1978).

[23] S. Seletskiy et al., Effect of beam-beam kick on electron beam quality in first bunched electron cooler, in Proceedings of IPAC2019, Melbourne, Australia (JACoW Publishing, 2019).

[24] S. Seletskiy et al., Absolute Energy Measurement of the LEReC Electron Beam, in Proceedings of NAPAC16, Chicago, IL (JACoW Publishing, 2016).

[25] F. Carlier, Radiative recombination detection to monitor electron cooling conditions during low energy RHIC operations, in Proceedings of IPAC2016, Busan, Korea (JACoW Publishing, 2016).

[26] S.Seletskiy, How to measure energy of LEReC electron beam with magnetic spectrometer, BNL Report No. BNL112105-2016-IR, 2016.

[27] H. Song et al., High-Precision Magnetic Field Measurement and Mapping of the LEReC 180 Bending Magnet Using Very Low Field NMR Probe, in Proceedings of IMMW21, Grenoble, France, 2019 (to be published). 
[28] T. Miller et al., Low field NMR probe commissioning in LEReC energy spectrometer, in Proceedings of IBIC2017, Grand Rapids, MI (JACoW Publishing, 2017).

[29] M. Bell and J. S. Bell, Capture of cooling electrons by cool protons, Part. Accel. 12, 49 (1981).
[30] G. Robert-Demolaize, A. Drees, and Y. Luo, Simulations of low energy Au78+ losses in RHIC, in Proceedings of NAPAC19, Lansing, MI, USA (to be published).

[31] A Drees et al., Report on LEReC recombination monitor APEX study June 15 2016, BNL Report No. BNL-1133842016-Tech, 2016. 